\title{
MICROLOCAL REGULARITY ON STEP TWO NILPOTENT LIE GROUPS
}

\author{
by KENNETH G. MILLER
}

(Received 9th April 1986)

\section{Introduction}

A necessary and sufficient condition for a homogeneous left invariant partial differential operator $P$ on a nilpotent Lie group $G$ to be hypoelliptic is that $\pi(P)$ be injective in $\mathscr{S}_{\pi}$ for every nontrivial irreducible unitary representation $\pi$ of $G$. This was conjectured by Rockland in [18], where it was also proved in the case of the Heisenberg group. The necessity of the condition in the general case was proved by Beals [2] and the sufficiency by Helffer and Nourrigat [4]. In this paper we present a microlocal version of this theorem when $G$ is step two nilpotent. The operator may be homogeneous with respect to any family of dilations on $G$, not just the natural dilations. We may also consider pseudodifferential operators as well as partial differential operators.

Throughout the paper $G$ is assumed to be a connected, simply connected nilpotent Lie group and is step two nilpotent unless otherwise stated. Let $\mathscr{G}$ be the Lie algebra of $G$, let $\mathscr{G}^{*}$ be the dual of $\mathscr{G}$ and $\dot{G}^{*}=\mathscr{G}^{*}-\{0\}$. If $\Omega$ is an open subset of $G$, a pseudodifferential operator $P$ on $\Omega$ is said to be microhypoelliptic (or microlocally hypoelliptic) at $(g, \xi) \in \Omega \times \dot{\mathscr{G}}^{*}$ if $(g, \xi) \in W F(P u)$ for every $u \in \mathscr{D}^{\prime}(\Omega)$ for which $(g, \xi) \in W F(u)$. In this paper, for the most part, rather than considering the usual wave front set, $W F(u)$, we will consider a variant of the wave front set, $W F_{\delta}(u)$, based on sets which are conic with respect to some family $\delta=\left\{\delta_{r}: r>0\right\}$ of dilations on $G$, and the corresponding notion of $\delta$-microhypoellipticity. On $\mathbb{R}^{n}$ similar types of wave front have been considered by Lascar [8] and Parenti and Rodino [15], among others. On a Lie group the definitions need to be modified somewhat to take into account the action of $G$ on $\mathscr{G}^{*}$. Details are in Section 2 .

Given $\xi \in \mathscr{G}^{*}$ let $\mathcal{O}_{\xi}$ be the orbit of $\xi$ under the coadjoint action of $G$ on $\mathscr{G}^{*}$ and let $\pi_{\xi}$ be the irreducible unitary representation corresponding to $\xi$ in the Kirillov theory. Let $\tilde{G}^{*}$ be the set of $\xi \in \mathscr{G}^{*}$ for which $\operatorname{dim} \mathcal{O}_{\xi}$ is maximal. $\mathscr{G}^{*}$ is an open dense subset of $\mathscr{G}^{*}$. If $\delta$ is a family of dilations on $G$, a subset $\Gamma$ of $\dot{G}^{*}$ is said to be a $\delta$-cone if it is invariant under $\delta$. $\Gamma \subset \mathscr{G}^{*}$ is said to be $G$-invariant if it is invariant under the coadjoint action of $G$ on $\mathscr{G}^{*}$, i.e. if $\Gamma$ is a union of orbits. Let $\Gamma_{\xi}$ be the smallest $G$-invariant $\delta$-cone containing $\xi$.

If $P$ is a left invariant operator which is homogeneous with respect to the dilations $\delta$, then the injectivity of $\pi_{\xi}(P)$ implies the injectivity of $\pi_{\eta}(P)$ for all $\eta \in \Gamma_{\xi}$. One might therefore expect that the injectivity of $\pi_{\xi}(P)$ is related to $\delta$-microhypoellipticity on $\Gamma_{\xi}$. Rather than considering just $\Gamma_{\xi}$ it is helpful to consider $\Gamma_{\xi}=\left(\right.$ closure of $\left.\Gamma_{\xi}\right)-\{0\}$. We note (Proposition 3.2) that $\partial \Gamma_{\xi}=\bar{\Gamma}_{\xi}-\Gamma_{\xi} \subset[\mathscr{G}, \mathscr{G}]^{\perp}$, (for standard dilations $\partial \Gamma_{\xi}=R_{\xi}^{\perp}$, where $R_{\xi}$ is the radical of the bilinear form associated with $\xi$ ), and hence $\pi_{\eta}$ is a one dimensional representation for $\eta \in \partial \Gamma_{\xi}$. The following two theorems are the main results of the paper. In both $G$ is a step two group with a family of dilations $\delta$. 
Theorem. Let $P$ be a left invariant pseudodifferential operator on $G$ which is homogeneous with respect to $\delta$. If $P$ is $\delta$-microhypoelliptic at $(e, \eta)$ for every $\eta \in \Gamma_{\xi}$, then $\pi_{\xi}(P)$ is injective in $\mathscr{S}_{n}$.

Theorem. Let $P$ be a pseudodifferential operator on $\Omega \subset G$. Let $P_{g}^{0}$ be the principal part of the invariant operator $P_{g}$ obtained by "freezing the coefficients" of $P$ at $g$. Let $\Gamma$ be an open $\delta$-conic subset of $\Omega \times \widetilde{G}^{*}$. If, for every $(g, \xi) \in \Gamma, \pi_{\eta}\left(P_{g}^{0}\right)$ is injective for all $\eta \in \Gamma_{\xi}$, then $P$ is $\delta$-microhypoelliptic on $\Gamma$.

The first theorem is proved in Section 3, the second is stated more precisely and proved in Section 4. These results were announced in slightly less generality in [14].

The second result can be improved somewhat when the dilations are natural dilations on $G$, i.e. when $\delta_{r} x=r^{2} x$ for $x \in[\mathscr{G}, \mathscr{G}]$ and $\delta_{r} x=r x$ for $x$ in some supplement $\mathscr{G}_{1}$ of $[\mathscr{G}, \mathscr{G}]$. In Corollary 4.5 it is shown that for natural dilations in order to prove $\delta$ microhypoellipticity near $\left(g_{0}, \xi_{0}\right)$ it is not necessary to assume injectivity of $\pi_{\xi}\left(P_{g}^{0}\right)$ for all $(g, \xi)$ in a $\delta$-conic neighborhood of $\left(g_{0}, \zeta_{0}\right)$, but simply to assume the injectivity of $\pi_{\eta}\left(P_{g_{0}}^{0}\right)$ for all $\eta \in \bar{\Gamma}_{\xi_{0}}$. This generalizes a result proved by Grigis [3] for operators of order 2. Corollary 4.6 gives sufficient conditions for microhypoellipticity with respect to standard conic sets.

At least for partial differential operators the parametrix construction of Melin [10] shows that if $P$ is left invariant and homogeneous on a nilpotent Lie group $G$ (with no restriction on the nilpotence step) and if $\pi(P)$ is injective for all nontrivial irreducible unitary representations $\pi$ of $G$, then $P$ is globally microhypoelliptic in the standard sense, i.e. microhypoelliptic at $(g, \xi)$ for all $g \in G$ and $\xi \in \dot{G}^{*}$. The parametrix construction in [10] makes use of global a priori estimates proved by Helffer and Nourrigat [4] under the assumption of the injectivity of $\pi(P)$ for all nontrivial $\pi$. In order to construct a microlocal parametrix under the weaker hypotheses stated above, we use a different method. The construction, which is a refinement of that used in [13], makes use of the fact that the calculus for invariant operators on a step two group "fibres" over the orbits and the orbit level calculus is the Weyl calculus [12]. This allows us to construct the parametrix on the orbits individually in terms of the symbols of the inverses of the operators $\pi(P)$.

\section{Dilations and pseudodifferential operators}

A family of dilations on $\mathscr{G}$ is a continuous one parameter family $\delta=\left\{\delta_{r}: r>0\right\}$ of simultaneously diagonalizable automorphisms of $\mathscr{G}$ with positive eigenvalues such that $\delta_{r} \delta_{s}=\delta_{r s}$ for all $r, s>0$ and such that $\lim _{r \rightarrow 0} \delta_{r} x=0$ for all $x \in \mathscr{G}$. For each $r$ define $\delta_{r}: G \rightarrow G$ by $\delta_{r} g=\exp \delta_{r} \log g$ and define $\delta_{r}: \mathscr{G}^{*} \rightarrow \mathscr{G}^{*}$ to be the transpose of $\delta_{r}: \mathscr{G} \rightarrow \mathscr{G}$.

If $\mathscr{B}=\left\{e_{1}, \ldots, e_{n}\right\}$ is a basis of eigenvectors for $\left\{\delta_{r}: r>0\right\}$, then there are $\mu_{j}>0$ such that

$$
\delta_{r} e_{j}=r^{\mu_{j}} e_{j}
$$

Without loss of generality we may assume that $\min \mu_{j}=1$. Let $\bar{\mu}=\max \mu_{j}$. It can be easily shown (Lemma 1.2 of [11]) that there is a set $S=\left\{e_{1}, \ldots, e_{N}\right\}$ of linearly 
independent eigenvectors for $\left\{\delta_{r}: r>0\right\}$ which generate $\mathscr{G}$ and such that $\mathscr{G}_{1}=\operatorname{span} S$ intersects $\mathscr{G}_{2}=[\mathscr{G}, \mathscr{G}]$ trivially. Let $\left\{e_{N+1}, \ldots, e_{n}\right\}$ be a basis for $\mathscr{G}_{2}$ chosen so that each $e_{k}, k>N$, is a multiple of $\left[e_{i}, e_{j}\right]$ for some $i<j \leqq N$. Since $\left\{\delta_{r}: r>0\right\}$ is a family of automorphisms, each $e_{k}, k>N$, is also an eigenvector for $\delta_{r}$. If the numbers $\gamma_{i j}^{k}$ are defined by

$$
\left[e_{i}, e_{j}\right]=\sum \gamma_{i j}^{k} e_{k}
$$

and if the numbers $\mu_{j}$ are defined by (1.1), then

$$
\gamma_{i j}^{k} \neq 0 \text { implies } \mu_{i}+\mu_{j}=\mu_{k} .
$$

For $x \in \mathscr{G}$ let $|x|=\left(x_{1}^{2}+\cdots+x_{n}^{2}\right)^{1 / 2}$, where $\left(x_{1}, \ldots, x_{n}\right)$ are the coordinates of $x$ with respect to the basis $\left\{e_{1}, \ldots, e_{n}\right\}$. By replacing each $e_{k}, N<k \leqq n$, by $c e_{k}$ for sufficiently large $c$ we may assume that

$$
|[x, y]| \leqq|x||y|, \text { for all } x \text { and } y \text { in } \mathscr{G} .
$$

We fix a basis $\mathscr{B}=\left\{e_{1}, \ldots, e_{n}\right\}$ for $\mathscr{G}$ having the properties just described. Coordinates and norms on $\mathscr{G}$ and $G^{*}$ will always be with respect to this basis or its dual $\left\{e_{1}^{*}, \ldots, e_{n}^{*}\right\}$. If $\alpha$ is a multi-index, let $\mu \alpha=\sum \mu_{j} \alpha_{j}$.

For $\xi \in \mathscr{G}^{*}-\{0\}$, define $[\xi]$ by $[\xi]=r$ if $\left|\delta_{r}^{-1} \xi\right|=1$. Note that in terms of the chosen coordinate system

$$
[\xi] \approx \sum\left|\xi_{j}\right|^{1 / \mu_{j}}
$$

Let $\chi: \mathscr{G}^{*} \rightarrow \mathbb{R}$ be a smooth function such that $\chi(\xi) \approx[\xi]+1$.

Definition. Let $\delta$ be a family of dilations on $\mathscr{G}$ and let $m \in \mathbb{R} . S^{m}\left(\mathscr{G}^{*}, \delta\right)$ is the set of $p \in C^{\infty}\left(\mathscr{G}^{*}\right)$ such that for every multi-index $\alpha$ there is a $C_{\alpha}$ such that

$$
\left|D^{\alpha} p(\xi)\right| \leqq C_{\alpha} \chi(\xi)^{m-\mu \alpha}
$$

for all $\xi \in \mathscr{G}^{*}$. If $\Omega$ is an open subset of $G, S^{m}\left(\Omega \times \mathscr{G}^{*}, \delta\right)$ is the set of $p \in C^{\infty}\left(\Omega \times \mathscr{G}^{*}\right)$ such that for every compact $K \subset \Omega_{1}=\log \Omega$ and all multi-indices $\alpha$ and $\beta$ there is a $C_{\alpha \beta K}$ such that

$$
\left|D_{x}^{\beta} D_{\xi}^{\alpha} p(\exp x, \xi)\right| \leqq C_{\alpha \beta K} \chi(\xi)^{m-\mu \alpha}, \text { for all }(x, \xi) \in K \times \mathscr{G}^{*}
$$

If $p \in S^{m}\left(\mathscr{G}^{*}, \delta\right)$, define the left invariant operator $P=\mathrm{Op}(p)$ by

$$
P u=u * F_{1}^{-1} p, \quad u \in \mathscr{P}(G)
$$

where * denotes convolution on $G$ and $F_{1}^{-1} p=F^{-1} p \circ \log , F: \mathscr{S}^{*}(\mathscr{G}) \rightarrow \mathscr{S}^{*}\left(\mathscr{G}^{*}\right)$ the 
Euclidean Fourier transform. If $p \in S^{m}\left(\Omega \times \mathscr{G}^{*}, \delta\right)$ define

$$
\begin{gathered}
P=\mathrm{Op}(p): \mathscr{D}(\Omega) \rightarrow \mathscr{E}(\Omega) \quad \text { by } \\
P u(g)=\mathrm{Op}\left(p_{g}\right) u(g),
\end{gathered}
$$

where $p_{g}(\xi)=p(g, \xi)$ and $\mathrm{Op}\left(p_{g}\right)$ is defined by $(1.7)$.

Note that if $p \in C^{\infty}\left(\mathscr{G}^{*}\right)$ is homogeneous of degree $m$ with respect to $\delta$ for large $\xi$, i.e. $p(\delta, \xi)=r^{m} p(\xi)$ for $|\xi| \geqq C$, then $p \in S^{m}\left(\mathscr{G}^{*}, \delta\right)$.

There are two asymptotic expansions which are important in the parametrix construction given in Section 4. The first is for the symbol $p \square q=F_{1}\left(F_{1}^{-1} p * F_{1}^{-1} q\right)$ of a product $\mathrm{Op}(p) \mathrm{Op}(q)$ where $p \in S^{m_{1}}\left(\mathscr{G}^{*}, \delta\right), q \in S^{m_{2}}\left(\mathscr{G}^{*}, \delta\right)$. Given $\xi \in \mathscr{G}^{*}$ let $\xi^{\prime}=\left.\xi\right|_{\mathscr{S}_{2}}$ and let

$$
h(\xi)=\left|\delta_{x(\xi)}^{-1} \xi^{\prime}\right| \text {. }
$$

Following Melin [9], given $m \in \mathbb{R}$ and $k \geqq 0$ we define $S^{m, k}\left(\mathscr{G}^{*}, \delta\right)$ to be the set of $p \in C^{\infty}\left(\mathscr{G}^{*}\right)$ such that

$$
\left|D^{\alpha} p(\xi)\right| \leqq C_{\alpha} h(\xi)^{\max \left\{k-\left|\alpha^{\prime}\right|, 0\right\}} \chi(\xi)^{m-\mu \alpha}, \quad \xi \in \mathscr{G}^{*} .
$$

Define the higher order brackets $\{p, q\}_{j}$ as in [12]. The following theorem can be proved using the Weyl Calculus of Hörmander [6] as in [12].

Theorem 1.1. Let $p \in S^{m_{1}, k_{1}}\left(\mathscr{G}^{*}, \delta\right), q \in S^{m_{2, k_{2}}}\left(\mathscr{G}^{*}, \delta\right)$. For any integer $J \geqq 0$,

$$
\begin{aligned}
& p \square q=\sum_{j<J}(i / 2)^{j}(j !)^{-1}\{p, q\}_{j}+r_{J} \\
& \text { where } r_{J} \in S^{m_{1}+m_{2,}, k_{1}+k_{2}+J}\left(\mathscr{G}^{*}, \delta\right) .
\end{aligned}
$$

The second asymptotic expansion, due to Michael Taylor [19], is for the symbol $p \# q$ of the product $\operatorname{Op}(p) \operatorname{Op}(q)$ where $p \in S^{m}\left(\Omega \times \mathscr{G}^{*}, \delta\right), q \in S^{k}\left(\Omega \times \mathscr{G}^{*}, \delta\right)$. If $\alpha$ is a multi-index let $t_{\alpha}(\xi)=\xi^{\alpha}, \xi^{\alpha}$ being defined in terms of the chosen coordinate system, and let $T^{\alpha}=\mathrm{Op}\left(t_{\alpha}\right)$. If $p \in S^{m}\left(\Omega \times \mathscr{G}^{*}, \delta\right)$, let $T_{g}^{\alpha} p$ refer to the function obtained by applying $T^{\alpha}$ to $p$ as a function on $\Omega$ as $\xi$ is held fixed. Define $p \square q q(g, \xi)=\left(p_{g} \square q_{g}\right)(\xi)$.

Theorem 1.2 ([19]). Let $p \in S^{m}\left(\Omega \times \mathscr{G}^{*}, \delta\right), q \in S^{k}\left(\Omega \times \mathscr{G}^{*}, \delta\right)$ with $\mathrm{Op}(q)$ properly supported. Then

$$
p \# q=\sum_{|\alpha|<s}\left(i^{\alpha} / \alpha !\right) D_{\xi}^{\alpha} p \bigsqcup T_{g}^{\alpha} q+r_{J}
$$

where $r_{J} \in S^{m+k-J}\left(\Omega \times \mathscr{G}^{*}, \delta\right)$.

Let $\xi \in \mathscr{G}^{*}$, let $\tilde{V}$ be a subspace of $\mathscr{G}$ maximally subordinate to $\xi, V$ a supplement to $\tilde{V}$ in $\mathscr{G}$, and let $\pi=\pi_{\xi, V, \nu}$ be the irreducible unitary representation of $G$ on $L^{2}(V)$ as defined in [4] or [13]. Let $\mathcal{O}_{\pi}$ be the orbit of the coadjoint action corresponding to $\pi$ in 
the Kirillov theory. Let $\psi_{\pi}$ be the symplectomorphism from $V \times V^{*}$ onto $\mathcal{O}_{\pi}$ defined in [12]. $\left(\psi_{\pi}\right.$ will sometimes be denoted $\left.\psi_{\xi}\right)$. If $p \in S^{m}\left(\mathscr{G}^{*}, \delta\right)$ and $P=\mathrm{Op}(p)$, define $\pi(P)$ to be the pseudodifferential operator on $V$ with Weyl symbol $p_{\pi}$ where

$$
p_{\pi}=p \circ \psi_{\pi}
$$

It was shown in [12] that $(a) \pi(P Q)=\pi(P) \pi(Q) ;(b) \pi(P)$ agrees with the usual definition of $\pi(P)$ when $P$ is an invariant differential operator and (c) if $p \in \mathscr{S}\left(\mathscr{G}^{*}\right)$ then

$$
\pi(P)=\pi\left(F_{1} p\right)
$$

Given $\xi \in \mathscr{G}_{2}^{*}$ let $d=d(\zeta)$ be the rank of the bilinear from $B_{\zeta}(x, y)=\langle\zeta,[x, y]\rangle$ on $\mathscr{G} \times \mathscr{G}$. As shown in [4] and [11], the irreducible unitary representations of $G$ can be parametrized by $\zeta \in \mathscr{G}_{2}^{*}$ and $\rho \in \mathbb{R}^{N-2 d}, N=\operatorname{dim} \mathscr{G}_{1}$ : Every irreducible unitary representation of $\mathscr{G}$ is equivalent to exactly one of the representations $\pi_{\rho \zeta}$ as defined in [11]. Given a family of dilations it is convenient to replace $\pi_{\rho \zeta}$ for $\zeta \neq 0$ by $\pi_{\rho \zeta_{0}} \circ \delta_{r}$ where $r=[\zeta]$ and $\zeta_{0}=\delta_{r}^{-1} \zeta$, for we then have

$$
\pi_{\rho \zeta}(P)=[\zeta]^{m} \pi_{\rho \zeta_{0}}(P)
$$

if $P$ is homogeneous with respect to $\delta$. We will let $p_{\rho \zeta}=p_{\pi}$ and $\mathcal{O}_{\rho \zeta}=\mathcal{O}_{\pi}$ where $\pi=\pi_{\rho \zeta}$.

\section{Microlocal analysis on $\boldsymbol{G}$}

If $k \in \mathbb{R}$ and $g \in G$, define $k g \in G$ by $k g=\exp (k \log g)$. For $a \in G$, define $\lambda^{a}: G \rightarrow G$ by $\lambda^{a} g=a^{-1} g$. We identify the tangent bundle $T G$ with $G \times \mathscr{G}$. If $G$ is step two nilpotent, then for any $a$ and $b$ in $G,\left(d \lambda^{a}\right)_{b}=\operatorname{Ad}\left(-\frac{1}{2} a\right): \mathscr{G} \rightarrow \mathscr{G}$. If $\phi: M \rightarrow N$ is a diffeomorphism let $\phi_{*}$ denote the naturally induced map $\phi_{*}: T^{*} M \rightarrow T^{*} N$. Since $G$ is step two nilpotent, if $a \in G, g \in G$ and $\xi \in \mathscr{G}^{*}$, then

$$
\lambda_{*}^{a}(g, \xi)=\left(a^{-1} g,\left(\operatorname{Ad} \frac{1}{2} a\right)^{*} \xi\right),
$$

since $\left(\operatorname{Ad}-\frac{1}{2} a\right)^{-1}=\operatorname{Ad} \frac{1}{2} a$.

Let $\mathscr{G}^{*}=\mathscr{G}^{*}-\{0\}$. Let $\left\{\delta_{r}: r>0\right\}$ be a family of dilations on $\mathscr{G}$. A subset $\Gamma$ of $\mathscr{G}^{*}$ is called a $\delta$-cone if $\xi \in \Gamma$ implies $\delta_{r} \xi \in \Gamma$ for all $r>0$. If $\Gamma$ is a $\delta$-cone, then $\Gamma$ will denote (closure $\Gamma$ )- $\{0\}$. The notation $\Gamma_{1} \subset \subset \Gamma_{2}$ for $\delta$-cones means $\Gamma_{1} \subset \Gamma_{2}$.

Definition. A set $\Gamma \subset G \times \dot{G}^{*}$ is $\delta$-conic if for every $g \in G,\left\{\left(\operatorname{Ad}_{2} \frac{1}{2} g\right)^{*} \xi:(g, \xi) \in \Gamma\right\}$ is a $\delta$ cone. In other words, $\Gamma$ is $\delta$-conic if and only if for all $g \in G, \lambda_{*}^{g}\left(\Gamma_{\theta}\right)$ is a $\delta$-cone in $T_{e}^{*} G \cong \mathscr{G}^{*}$, where $\Gamma_{g}$ is the fibre of $\Gamma$ over $g$.

Note that if $\Gamma \subset G \times \mathscr{G}^{*}$ is $\delta$-conic and $a \in G$, then $\lambda_{*}^{a} \Gamma$ is also $\delta$-conic. It should also be noted that if $\Gamma \subset \dot{G}^{*}$ is a $\delta$-cone and $a \in G$, then $(\mathrm{Add} a)^{*} \Gamma$ is not usually a $\delta$-coneeven in the case of the natural dilations on the Heisenberg group. That is why we did not define a set $\Gamma \subset G \times \dot{G}^{*}$ to be $\delta$-conic if $\Gamma_{g}$ is a $\delta$-cone for every $g$.

If $\omega \subset G$ and $\Gamma^{\prime} \subset \dot{G}^{*}$ is a $\delta$-cone, let $\omega^{*} \Gamma^{\prime}=\left\{(g, \xi): g \in \omega\right.$ and $\left.\left(\operatorname{Ad} \frac{1}{2} g\right)^{*} \xi \in \Gamma^{\prime}\right\}$. If $g \in G$, let 
$g^{*} \Gamma^{\prime}=\left\{\xi:\left(\operatorname{Ad} \frac{1}{2} g\right)^{*} \xi \in \Gamma^{\prime}\right\}$. Note that if $\Gamma \subset G \times \mathscr{G}^{*}$ is an open $\delta$-conic set and $\left(g_{0}, \xi_{0}\right) \in \Gamma$, then there is an open neighborhood $\omega$ of $g_{0}$ and an open $\delta$-cone $\Gamma^{\prime}$ containing $\left(\operatorname{Ad} \frac{1}{2} g_{0}\right)^{*} \xi_{0}$ such that $\omega^{*} \Gamma^{\prime} \subset \Gamma$. Let $F_{1} u=F(u \circ \exp )$ where $F$ is the Fourier transform.

Definition. Let $\Omega$ be an open subset of $G$, let $p r_{2}: \Omega \times \dot{\mathscr{G}}^{*} \rightarrow \dot{\mathscr{G}}^{*}$ be the projection and let $u \in \mathscr{D}^{\prime}(\Omega)$. Define $W F_{\delta}(u) \subset \Omega \times \dot{G}^{*}$ by $(a, \eta) \notin W F_{\delta}(u)$ if there is a $\delta$-conic neighborhood $\Gamma$ of $(a, \eta)$ and a $\phi \in C_{c}^{\infty}(\Omega), \phi \equiv 1$ in some neighborhood of $a$, such that $F_{1}(\phi u)$ is rapidly decreasing in $p r_{2} \Gamma$, i.e. for every $N$

$$
\left|F_{1}(\phi u)(\xi)\right| \leqq C_{N}(1+|\xi|)^{-N}, \quad \text { for } \xi \in p r_{2} \Gamma
$$

Definition. Let $p \in S^{m}\left(\Omega \times \mathscr{G}^{*}, \delta\right) . P=\mathrm{Op}(p)$ is said to be regularizing on an open $\delta$ conic set $\Gamma$ if given $(a, \eta) \in \Gamma$, there is a $\phi$ as in the preceding definition and an open $\delta$ cone $\Gamma^{\prime}$ with $\eta \in a^{*} \Gamma^{\prime} \subset \Gamma_{a}$ such that

$$
\left|D_{x}^{\alpha} D_{\xi}^{B} \phi(\exp x) p(\exp x, \xi)\right| \leqq C_{\alpha \beta N}(1+|\xi|)^{-N}
$$

for all $\xi \in \Gamma^{\prime}$. The complement of the union of all $\delta$-conic open sets on which $P$ is regularizing is denoted microsupp $p_{\delta}(P)$.

If $P$ is left invariant we sometimes refer to $\left\{\xi:(e, \xi) \in \operatorname{microsupp}_{\delta}(P)\right\} \subset \dot{G}^{*}$ as simply microsupp $_{\delta}(P)$.

Definition. Let $P \in O p S^{m}\left(\Omega \times \mathscr{G}^{*}, \delta\right)$. Let $\Gamma$ be a $\delta$-conic subset of $\Omega \times \mathscr{G}^{*} . P$ is said to be $\delta$-microhypoelliptic on $\Gamma$ if $W F_{\delta}(u) \cap \Gamma \subset W F_{\delta}(P u) \cap \Gamma$ for every $u \in \mathscr{D}^{\prime}(\Omega)$. Let $(g, \xi) \in \Omega \times \dot{G}^{*} . P$ is said to be $\delta$-microhypoelliptic at $(g, \xi)$ if it is $\delta$-microhypoelliptic on the smallest $\delta$-conic set containing $(g, \xi)$. P is said to be $\delta$-microhypoelliptic near $(g, \xi)$ if it is $\delta$-microhypoelliptic on a $\delta$-conic neighborhood of $(g, \xi)$.

If $a \in G$ and $P \in \mathrm{Op} S^{m}\left(\lambda^{a} \Omega \times \mathscr{G}^{*}, \delta\right)$ define $P^{a} \in \mathrm{Op} S^{m}\left(\Omega \times \mathscr{G}^{*}, \delta\right)$ by $P^{a} u=P\left(u \circ \lambda^{a^{-1}}\right) \circ \lambda^{a}$. Then $P^{a}=\mathrm{Op}\left(p^{a}\right)$ where $p^{a}(g, \xi)=p\left(\lambda^{a} g, \xi\right)$.

Proposition 2.1. Let $a \in G, u \in \mathscr{D}^{\prime}(\Omega)$ and $P \in \mathrm{OpS}^{m}\left(\lambda^{a} \Omega \times \mathscr{G}^{*}, \delta\right)$. Then

(a) $\lambda_{*}^{a} W F_{\delta}\left(u \circ \lambda^{a}\right)=W F_{\delta}(u)$;

(b) $\lambda_{*}^{a} \operatorname{microsupp}_{\delta}\left(P^{a}\right)=\operatorname{microsupp}_{\delta}(P)$;

(c) For any $\delta$-conic set $\Gamma, P^{a}$ is $\delta$-microhypoelliptic on $\Gamma$ if and only if $P$ is $\delta$ microhypoelliptic on $\lambda_{*}^{a} \Gamma$.

Proof. Since

$$
F_{1}\left(u \circ \lambda^{a}\right)(\xi)=e^{-i\langle\xi, \log a\rangle} F_{1} u\left(\operatorname{Ad} \frac{1}{2} a^{*} \xi\right)
$$

it follows that $F_{1}\left(\phi u \circ \lambda^{a}\right)$ is rapidly decreasing in $p r_{2} \Gamma$ if and only if $F_{1}(\phi u)$ is rapidly decreasing in $p r_{2} \lambda_{*}^{a} \Gamma$. This implies (a). The proof of (b) is trivial and (c) follows from (a).

Corollary 2.2. If $P \in \mathrm{Op} S^{m}\left(\mathscr{G}^{*}, \delta\right)$ and $P$ is $\delta$-microhypoelliptic at (near) $(e, \xi)$ for all 
$\xi \in \mathcal{O}$, where $\mathcal{O} \subset \dot{G}^{*}$ is some orbit, then $P$ is $\delta$-microhypoelliptic at (near) $(g, \xi)$ for all $g \in G$ and all $\xi \in \mathcal{O}$.

Corollary 2.3. If $P \in \mathrm{Op} S^{m}\left(\mathscr{G}^{*}, \delta\right)$ is $\delta$-microhypoelliptic at (near) $(g, \xi)$ for all $g \in G$, then $P$ is $\delta$-microhypoelliptic at (near) $(g, \eta)$ for all $g \in G$ and all $\eta \in \mathcal{O}_{\xi}$.

Proposition 2.4. Let $\Omega$ be an open subset of $G$ and $\Gamma$ an open $\delta$-conic subset of $\Omega \times \dot{G} *$. Let $P_{j} \in \mathrm{Op} S^{m_{j}}\left(\Omega \times \mathscr{G}^{*}, \delta\right)$ for $j=1,2$, with $P_{1}$ and $P_{2}$ either both properly supported or both left invariant. If microsupp $P_{1} \cap$ microsupp $_{\delta} P_{2} \cap \Gamma=\phi$, then $P_{1} P_{2}$ is regularizing on $\Gamma$.

Proof. First consider the case when $P_{1}$ and $P_{2}$ are left invariant. Then $\Gamma$ and microsupp $P_{j}$ may be regarded as $\delta$-cones in $\mathscr{G}^{*}$. Let $p_{j}$ be the symbol of $P_{j}$. If $p_{j} \in \mathscr{S}\left(\mathscr{G}^{*}\right)$, then

$$
\begin{aligned}
p_{1} \square p_{2}(\eta) & =\iint_{\xi \xi \mathscr{G}} e^{i\langle\eta-\xi, x\rangle} p_{1}(\xi) p_{2}\left(\eta+\operatorname{ad} \frac{1}{2} x^{*} \eta\right) d x d \xi \\
& =\iint_{\mathscr{S} * \mathscr{G}} e^{i\langle\eta-\xi, x\rangle} p_{1}\left(\eta-\operatorname{ad} \frac{1}{2} x^{*} \eta\right) p_{2}(\xi) d x d \xi .
\end{aligned}
$$

We shall show that there exist $C_{N}$ such that

$$
\left|\left(p_{1} \square p_{2}\right)(\eta)\right| \leqq C_{N}\left(1+|\eta|^{-N}\right) \text { for all } \eta \in \Gamma,
$$

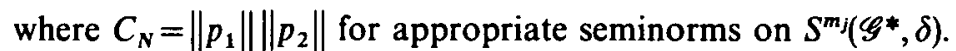

For $j=1,2$ there exist open $\delta$-cones $\Gamma_{j}, \Gamma_{j}^{\prime}, \Gamma_{j}^{\prime \prime}$ and a $c>0$ such that microsupp $P_{j} \subset \Gamma_{j}$, microsupp $_{\delta} P_{j} \cap \Gamma_{j}^{\prime}=\phi, \Gamma_{j} \cup \Gamma_{j}^{\prime}=\dot{G}^{*}, \Gamma_{1}^{\prime \prime} \cup \Gamma_{2}^{\prime \prime}=\Gamma$ and if $\xi \in \Gamma_{j}, \eta \in \Gamma_{j}^{\prime \prime}$, then $[\xi-\eta] \geqq c[\eta]$.

If $\eta \in \Gamma_{1}^{\prime \prime}$ we apply integration by parts to the first formula in (2.2) to obtain

$$
\begin{array}{r}
p_{1} \bigsqcup p_{2}(\eta)=\iint e^{i\langle\eta-\xi, x\rangle}\langle x\rangle^{-2 M}\left(I-\Delta_{\xi}\right)^{M}\left(p_{1}(\xi)\langle\eta-\xi\rangle^{-2 M}\right) . \\
\left(I-\Delta_{x}\right)^{M} p_{2}\left(\eta+\operatorname{ad} \frac{1}{2} x^{*} \eta\right) d x d \xi
\end{array}
$$

for all $M>0$, where $\langle x\rangle=\left(1+|x|^{2}\right)^{1 / 2}$. For sufficiently large $M,(2.4)$ is valid for all $p_{j} \in S^{m_{j}}\left(\mathscr{G}^{*}, \delta\right), j=1,2$. Note that $\left\langle\eta+\operatorname{ad} x^{*} \eta\right\rangle \leqq C\langle x\rangle\langle\eta\rangle$. Hence if $M$ is sufficiently large

$$
\left|p_{1} \bigsqcup p_{2}(\eta)\right| \leqq C\langle\eta\rangle^{\left|m_{2}\right|} \int\left|\left(I-\Delta_{\xi}\right)^{M}\left(p_{1}(\xi)\langle\eta-\xi\rangle^{-2 M}\right)\right| d \xi
$$

for $\eta \in \Gamma_{1}^{\prime \prime}$. We estimate this integral over $\Gamma_{1}$ and $\Gamma_{1}^{\prime}$ separately. Since $P_{1}$ is regularizing on $\Gamma_{1}^{\prime}$, it follows from Peetre's inequality that

$$
\int_{\Gamma_{1}}\left|\left(I-\Delta_{\xi}\right)^{M}\left(p_{1}(\xi)\langle\eta-\xi\rangle^{-2 M}\right)\right| d \xi \leqq C_{M}\langle\eta\rangle^{-2 M} .
$$

If $\xi \in \Gamma_{1}$, then $\langle\eta-\xi\rangle \geqq c(1+[\eta-\xi]) \geqq c(1+[\eta]) \geqq c\langle\eta\rangle^{1 / \bar{\mu}}$, and

$$
\left|\left(I-\Delta_{\xi}\right)^{M}\left(p_{1}(\xi)\langle\eta-\xi\rangle^{-2 M}\right)\right| \leqq C_{M} \chi(\eta)^{m_{1}}\langle\eta-\xi\rangle^{-2 M+\mid m_{1} !} .
$$


Thus

$$
\int_{\Gamma_{1}}\left|\left(I-\Delta_{\xi}\right)^{M}\left(p_{1}(\xi)\langle\eta-\xi\rangle^{-2 M}\right)\right| d \xi \leqq C_{M}\langle\eta\rangle^{2\left|m_{1}\right|+n+1-2 M / \bar{\mu}}
$$

if $M$ is sufficiently large. Since $\Gamma_{1} \cup \Gamma_{1}^{\prime}=\dot{G}^{*}$, (2.3) is verified for $\eta \in \Gamma_{1}^{\prime \prime}$. To prove (2.3) for $\eta \in \Gamma_{2}^{\prime \prime}$ apply a similar argument using the second formula in (2.2). By using (4.1) below, it follows that

$$
\left|D^{a}\left(p_{1} \bigsqcup \dot{p_{2}}\right)(\eta)\right| \leqq C_{a N}\left(1+|\eta|^{-N}\right) \text { for } \eta \in \Gamma_{1},
$$

which proves the proposition when $P_{1}$ and $P_{2}$ are left invariant.

For general $P_{1}$ and $P_{2}$ the preceding argument shows that for every $\alpha, \operatorname{Op}\left(D_{\xi}^{\alpha} p_{1} \bigsqcup T_{g}^{\alpha} p_{2}\right)$ is regularizing on $\Gamma$. By Theorem $1.2 P_{1} P_{2}$ is regularizing on $\Gamma$ if $P_{1}$ and $P_{2}$ are properly supported.

Propositions 2.5 and 2.6 now follow by standard arguments.

Proposition 2.5. Let $\Omega$ be an open subset of $G, u \in \mathscr{D}^{\prime}(\Omega)$. Then $(a, \eta) \notin W F_{\delta}(u)$ if and only if there is a $\delta$-conic neighborhood $\Gamma$ of $(a, \eta)$ such that $P u \in C^{\infty}(\Omega)$ for every properly supported $P \in \mathrm{Op}^{m}\left(\Omega \times \mathscr{G}^{*}, \delta\right)$ for which microsupp ${ }_{\delta} P \subset \Gamma$.

Proposition 2.6. If $Q \in \mathrm{Op} S^{m}\left(\Omega \times \mathscr{G}^{*}, \delta\right)$ and $u \in \mathscr{D}^{\prime}(\Omega)$, then

$$
W F_{\delta}(Q u) \subset W F_{\delta}(u) \cap \operatorname{microsupp}_{\delta} Q .
$$

\section{A necessary condition for $\delta$-microhypoellipticity}

If $\xi \in \dot{G}^{*}=\mathscr{G}^{*}-\{0\}$, let $\Gamma_{\xi}$ be the smallest $G$-invariant $\delta$-cone containing $\xi$. Let $\Gamma_{\xi}=$ (closure of $\left.\Gamma_{\xi}\right)-\{0\}$, and $\partial \Gamma_{\xi}=\Gamma_{\xi}-\Gamma_{\xi}$. Later in this section an algebraic description of $\partial \Gamma_{\xi}$ will be given, but our primary concern is the following necessary condition for $\delta$ microhypoellipticity on $\bar{\Gamma}_{\xi}$.

Theorem 3.1. Let $G$ be a step two nilpotent Lie group with a family of dilations $\delta$. Let $p \in C^{\infty}\left(\mathscr{G}^{*}\right)$ be homogeneous of degree $m$ with respect to $\delta$ for large $\xi$. Let $\xi \in \dot{G}^{*}$. If $P=\mathrm{Op}(p)$ is $\delta$-microhypoelliptic at $(e, \eta)$ for every $\eta \in \Gamma_{\xi}$, then $\pi_{\xi}(P)$ is injective in $\mathscr{S}_{\pi_{\xi}}$.

Proof. Suppose there is a $v \in \mathscr{S}_{\pi}, v \neq 0$, such that $\pi(P) v=0$, where $\pi=\pi_{\xi}$. Define the function $u(g)=(\pi(g) v, v)$ where (,) denotes the $L^{2}$ inner product. For all $r>0$ let $u_{r}=u \circ \delta_{r}, \pi_{r}=\pi \circ \delta_{r}$ and let $\mathcal{O}_{r}$ be the orbit corresponding to $\pi_{r}$. Then $u_{r}(g)=\left(\pi_{r}(g) v, v\right)$ for all $r>0$. If $p \in \mathscr{S}\left(\mathscr{G}^{*}\right)$, then

$$
\begin{aligned}
P u_{r}(g) & =u_{r} * F_{1}^{-1} p(g) \\
& =\int \pi_{r}(g) \pi_{r}(h) F_{1}(p)(h) v(t) \overline{v(t)} d h d t \\
& =\left(\pi_{r}(P) v, \pi_{r}\left(g^{-1}\right) v\right)
\end{aligned}
$$


by (1.11). By an approximation argument $P u_{r}(g)=\left(\pi_{r}(P) v, \pi_{r}\left(g^{-1}\right) v\right)$ for all $p \in S^{m}\left(\mathscr{G}^{*}, \delta\right)$. By redefining $p$ on a set with compact support we may assume that $p\left(\delta_{r} \eta\right)=r^{m} p(\eta)$ for all $\eta \in \mathcal{O}_{\xi}$ and all $r \geqq 1$. It follows that $\pi_{r}(P)=r^{m} \pi(P)$ for all $r \geqq 1$. Therefore $P u_{r}=0$ for all $r \geqq 1$.

Let $\Gamma_{1} \supset \supset \Gamma_{2} \supset \supset \ldots$ be a sequence of open $\delta$-cones containing $\Gamma_{\xi}$ such that $\cap \Gamma_{j}=\Gamma_{\xi}$, and let $\left\{\phi_{j}\right\}$ be a sequence of functions in $C_{0}^{\infty}(G)$ such that $e \in \operatorname{supp} \phi_{j+1} \subset \subset \operatorname{supp} \phi_{j}$ and $\varphi_{j} \geqq 0$. Let $C_{b}^{0}(G)$ denote the space of continuous bounded functions on $G$ with the supremum norm. Let $R_{j}=\left\{u \in C_{b}^{0}(G): F_{1}\left(u \phi_{j}\right)\right.$ is rapidly decreasing on $\left.\Gamma_{j}\right\} . R_{j}$ is a Frechet space with the seminorms

$$
\sigma_{k j}(u)=\sup \left\{\langle\eta\rangle^{k}\left|F_{1}\left(\phi_{j} u\right)(\eta)\right|: \eta \in \Gamma_{j}\right\},
$$

together with the $C_{b}^{0}(G)$ norm. It follows from the proof of Lemma 8.1.1 of [5] that $R_{j} \subset R_{j+1}$ and that the inclusion is continuous. Thus $R\left(\Gamma_{\xi}\right)=\cup R_{j}$ can be given the corresponding $L F$ topology.

Since $P$ is $\delta$-microhypoelliptic at $(e, \eta)$ for every point of $\Gamma_{\xi}$ and since $\left\{\eta \in \Gamma_{\xi}:[\eta]=1\right\}$ is compact, it follows that $\left\{u \in C_{b}^{0}(G): P u=0\right\} \subset R\left(\Gamma_{\xi}\right)$. The closed graph theorem implies that the inclusion is continuous. The set $\left\{u_{r}: r \geqq 1\right\}$ is a bounded subset of $\left\{u \in C_{b}^{0}(G)\right.$ : $P u=0\}$ and hence is a bounded subset of $R\left(\Gamma_{\xi}\right)$. Thus there is a $j$ such that $\left\{u_{r}: r \geqq 1\right\}$ is contained and bounded in $R_{j}$. Let $\phi=\phi_{j}$. Then for every $k>0$, there is a $C_{k}$ such that

$$
\left|F_{1}\left(\phi u_{r}\right)(\eta)\right| \leqq C_{k}\langle\eta\rangle^{-k}
$$

for all $\eta \in \bar{\Gamma}_{\xi}$ and all $r \geqq 1$.

Given $\eta \in^{\mathscr{G}} \mathscr{G}^{*}$ define $e_{\eta} \in C^{\infty}(G)$ by $e_{\eta}(\exp x)=e^{-i\langle\eta, x\rangle}$. Note that

$$
F_{1}\left(\phi u_{r}\right)(\eta)=\left(\int e^{-i \eta x} \phi(\exp x) \pi_{r}(\exp x) v d x, v\right)=\left(\pi_{r}\left(e_{\eta} \phi\right) v, v\right) .
$$

By (1.11), if $\phi \in C_{0}^{\infty}(G)$, then $\pi(\phi)$ is a pseudodifferential operator on $\mathbb{R}^{d}$ with Weyl symbol $F_{1}^{-1}(\phi) \circ \psi_{\pi}$. Since $F_{1}^{-1}\left(e_{\eta} \phi\right)(\zeta)=F_{1}^{-1} \phi(\zeta-\eta)$,

$$
\int_{O_{\pi}}\left(\pi\left(e_{\eta} \phi\right) v, v\right) d \eta=\int_{\mathbb{R}^{3 d} \int_{O_{\pi}}} e^{i(t-s) \tau} F_{1}^{-1} \phi\left(\psi_{\pi}((t+s) / 2, \tau)-\eta\right) v(s) \overline{v(t)} d \eta d s d t d \tau .
$$

For fixed $t, s$ and $\tau$, as $\eta$ varies over $\mathcal{O}_{n}, \psi_{\pi}((t+s) / 2, \tau)-\eta$ varies over $T \mathcal{O}_{\pi}$, the linear space parallel to $\mathcal{O}_{\pi}$. Thus by the Plancherel Theorem

$$
\int_{O_{x}}\left(\pi\left(e_{\eta} \phi\right) v, v\right) d \eta=\|v\|^{2} \int_{O_{x}} F_{1}^{-1} \phi(\eta) d \eta
$$

where $\|v\|$ is the $L^{2}$ norm of $v$. Assuming that the value of $\phi(\exp x)$ depends only on $|x|$, Lemma 3.3 below implies

$$
\int_{T \sigma_{z_{r}}} F_{1}^{-1} \phi(\eta) d \eta=\int_{R(\delta, \xi)} \phi(\exp x) d x=c
$$

where $c>0$ is independent of $r$, since the subspaces $R\left(\delta_{r} \xi\right)$ have the same dimension for 
all $r>0$. Thus $\int_{\sigma_{r}} F_{1}\left(\phi u_{r}\right)(\eta) d \eta=c$ is independent of $r$ and positive. But by (3.1),

$$
\left|\int_{0} F_{1}\left(\phi u_{r}\right)(\eta) d \eta\right| \leqq C \int_{0_{r}}\langle\eta\rangle^{-k} d \eta \rightarrow 0 \quad \text { as } \quad r \rightarrow \infty,
$$

if $k$ is sufficiently large. This contradiction implies that $\pi_{\xi}(P)$ is injective in $\mathscr{S}_{\pi}$.

We now give a more explicit description of $\partial \Gamma_{\xi}$. Let $R(\xi)=\left\{x \in \mathscr{G}_{1}:\langle\xi,[x, y]\rangle=0\right.$ for all $y \in \mathscr{G}\}$. Let $2 d(\xi)$ be the codimension of $R(\xi)$ in $\mathscr{G}_{1}$. If $\delta$ is a family of dilations, then $d\left(\delta_{r} \xi\right)=d(\xi)$ for all $r>0$. For fixed $\xi \in \dot{G}^{*}$, the map $\phi: r \rightarrow R\left(\delta_{r} \xi\right)$ is an algebraic function from $(0, \infty)$ into the Grassmann manifold $G R_{2 d}\left(\mathscr{G}_{1}\right)$ of subspaces of $\mathscr{G}_{1}$ of codimension $2 d=2 d(\xi)$. Since $G R_{2 d}\left(\mathscr{G}_{1}\right)$ is compact and $\phi$ is an algebraic function of one variable, $\phi$ extends to a continuous function $\phi:[0, \infty) \rightarrow G R_{2 d}\left(\mathscr{G}_{1}\right)$. Let $R_{0}(\xi)=$ $\phi(0)$, i.e. $R_{0}(\xi)=\lim _{r \rightarrow 0} R\left(\delta_{r} \xi\right)$.

Proposition 3.2. Let $G$ be a step two nilpotent Lie group with a family of dilations $\delta$. If $\xi \in \dot{G}^{*}$, then $\partial \Gamma_{\xi}=R_{0}(\xi)^{\perp}-\{0\}$. In particular, $\partial \Gamma_{\xi} \subset \mathscr{G}_{1}^{*}$.

Lemma 3.3. Define $\phi_{\xi}: \mathscr{G}_{1} \rightarrow \mathscr{G}_{1}^{*}$ by $\phi_{\xi}(x)=\operatorname{ad} x^{*} \xi$. Let $V \subset \mathscr{G}_{1}$ be a subspace such that $V \oplus R(\xi)=\mathscr{G}_{1}$. Then $\phi_{\xi}$ is a bijection of $V$ onto $R(\xi)^{\perp}$. Consequently the linear space $7 \mathcal{O}_{\xi}$ parallel to $\mathcal{O}_{\xi}$ is $R(\xi)^{\perp}$.

Proof. Clearly, the range of $\phi_{\xi}$ is contained in $R(\xi)^{\perp} \cong V^{*}$. Also $\left.\phi_{\xi}\right|_{V}$ is injective, therefore by a dimension argument the range of $\phi_{\xi}$ equals $R(\xi)^{\perp}$. The last statement follows from the observations that $T \mathscr{O}_{\xi}=\left\{\operatorname{ad} x^{*} \xi: x \in \mathscr{G}_{1}\right\}$.

Proof of Proposition 3.2. Write $\xi=\eta+\zeta$, where $\eta \in \mathscr{G}_{1}^{*}$ and $\zeta \in \mathscr{G}_{2}^{*}$. If $\zeta=0$, then $\partial \Gamma_{\xi}=\phi=R_{0}(\xi)^{\perp}-\{0\}$, so we may assume $\zeta \neq 0$.

Suppose $\xi_{j} \in \Gamma_{\xi}$ and $\lim \xi_{j}=\xi_{0}$. Then

$$
\xi_{j}=\delta_{r_{j}} \xi+\delta_{r_{j}} \text { ad } x_{j}^{*} \xi=\delta_{r_{j}} \eta+\delta_{r_{j}} \text { ad } x_{j}^{*} \zeta+\delta_{r_{j}} \zeta,
$$

where $\delta_{r_{j}} \eta+\delta_{r_{j}}$ ad $x_{j}^{*} \zeta \in \mathscr{G}_{1}^{*}$ and $\delta_{r_{j}} \zeta \in \mathscr{G}_{2}^{*}$. Since $\delta_{r_{j}} \zeta$ converges, $r_{j}$ must converge to $r_{0} \geqq 0$. Suppose $r_{0} \neq 0$. Then $\delta_{r_{j}}$ ad $x_{j}^{*} \zeta \rightarrow \eta^{\prime} \in \mathscr{G}_{1}^{*}$, and hence ad $x_{j}^{*} \zeta=\operatorname{ad} x_{j}^{*} \xi \rightarrow \delta_{r_{0}}^{-1} \eta^{\prime}$. Let $V$ and $\phi_{\xi}$ be as in Lemma 3.3. Since ad $x_{j}^{*} \xi \in R(\xi)^{\perp}$, we may assume that $x_{j} \in V$. Since $\phi_{\xi}$ is a linear bijection, the convergence of $\phi_{\xi}\left(x_{j}\right)$ implies that $x_{j} \rightarrow x_{0} \in V$. Thus $\xi_{0}=\delta_{r_{0}} \xi+$ $\delta_{r_{0}}$ ad $x_{0}^{*} \xi \in \Gamma_{\xi}$. Hence if $\xi_{0} \in \partial \Gamma_{\xi}$, then $r_{0}=0$. We may also write $\xi_{j}=\delta_{r_{j}} \xi+$ ad $y_{j}^{*}\left(\delta_{r_{j}} \xi\right)$, where ad $y_{j}^{*}\left(\delta_{r_{j}} \xi\right) \in R\left(\delta_{r_{j}} \xi\right)^{\perp}$. If $\xi_{0} \in \partial \Gamma_{\xi}$, then $r_{j} \rightarrow 0$, and hence $R\left(\delta_{r_{j}} \xi\right) \rightarrow R_{0}(\xi)$. Since $\delta_{\mathbf{r}_{j}} \xi \rightarrow 0$, it follows that $\xi_{0} \in R_{0}(\xi)^{\perp}$.

Conversely, if $\eta^{\prime} \in R_{0}(\xi)^{\perp}, \eta^{\prime} \neq 0$, choose $\eta_{r} \in R\left(\delta_{r} \xi\right)^{\perp}$ such that $\eta_{r} \rightarrow \eta^{\prime}$ as $r \rightarrow 0$. Then $\delta_{r} \xi+\eta_{r} \in \mathcal{O}_{\delta_{r} \xi} \subset \Gamma_{\xi}$. Hence $\eta^{\prime} \in \bar{\Gamma}_{\xi}$. Since $\mathscr{G}_{1}^{*} \cap \Gamma_{\xi}=\phi, \eta^{\prime} \in \partial \Gamma_{\xi}$.

Definition. If $G$ is a step two nilpotent Lie group a family of dilations $\left\{\delta_{r}: r>0\right\}$ is called natural if $\delta_{r} x=r^{2} x$ for all $x \in \mathscr{G}_{2}$ and $\delta_{r} x=r x$ for all $x \in \mathscr{G}_{1}$.

Corollary 3.4. If $G$ is a step two nilpotent group with natural dilations, or more generally, if $\mu_{j}=\bar{\mu}$ for all $j>N=\operatorname{dim} \mathscr{G}_{1}$, then $\partial \Gamma_{\xi}=R(\xi)^{\perp}-\{0\}$. 
Proof. Let $\zeta=\left.\xi\right|_{s_{2}}$. If $\mu_{j}=\bar{\mu}$ for all $j>N$, then $\delta_{r} \zeta=r^{\bar{\mu}} \zeta$, and $R\left(\delta_{r} \xi\right)=R(\xi)$ for all $r>0$. Hence $R_{0}(\xi)=R(\xi)$.

Note that for any family of dilations, given $\xi$ such that $\xi^{\prime}=\left.\xi\right|_{g_{2}} \neq 0$, there is a unique $s>0$ such that $\xi^{\prime \prime}=\lim _{r \rightarrow 0} r^{-s} \delta_{r} \xi^{\prime}$ exists and is non-zero. In fact $s=\min \left\{\mu_{j} ; j>N\right.$ and $\left.\xi_{j} \neq 0\right\}$. Since $R\left(r^{-s} \delta_{r} \xi^{\prime}\right)=R\left(\delta_{r} \xi^{\prime}\right), R_{0}(\xi) \subset R\left(\xi^{\prime \prime}\right)$ in all cases. If $d\left(\xi^{\prime \prime}\right)=d(\xi)$, then $R_{0}(\xi)=R\left(\xi^{\prime \prime}\right)$ and $\partial \Gamma_{\xi}=R\left(\xi^{\prime \prime}\right)^{\perp}-\{0\}$.

Let $G$ satisfy the condition $d(\xi)=d(\mathscr{G})$ for all $\xi \mathscr{G}_{1}^{*}$, i.e. $\mathscr{G}^{*}=\mathscr{G}^{*} \backslash \mathscr{G}_{1}^{*}$. Such groups were said to be typed $H$ in [13]. Then $R_{0}(\xi)=R\left(\xi^{\prime \prime}\right)$ as in the preceding paragraph. One might expect that $R_{0}(\xi)$ would depend continuously on $\xi \in \mathscr{G}^{*}$ in such a case. However, there are examples of dilations on the free step two nilpotent group on three generators (a six dimensional group of type $H$ ), where $R_{0}(\xi)$ does not depend continuously on $\xi \in \mathscr{G}^{*}$.

\section{A sufficient condition for microhypoellipticity}

Let $p \in S^{m}\left(\Omega \times \mathscr{G}^{*}, \delta\right)$, where $\Omega$ is an open subset of $G$. If $p$ can be written in the form $p=p^{0}+p^{1}$ where $p^{1} \in S^{m-\varepsilon}\left(\Omega \times \mathscr{G}^{*}, \delta\right)$ for some $\varepsilon>0$ and $p^{0}$ is homogeneous of degree $m$ with respect to $\delta$ in the $\xi$ variables, for large $\xi$, then $p^{0}$ is called the principal symbol of $p$.

Theorem 4.1. Let $G$ be a step two nilpotent Lie group with dilations $\delta$. Let $\Omega$ be an open subset of $G$ and let $p \in S^{m}\left(\Omega \times \mathscr{G}^{*}, \delta\right)$ have principal symbol $p^{0}$. Let $P=\mathrm{Op}(p)$ and $P_{g}^{0}=\mathrm{Op}\left(p_{g}^{0}\right)$ for $g \in \Omega$. Let $\Gamma$ be an open $\delta$-conic subset of $\Omega \times \mathscr{G}^{*}$. Assume that there is a $C$ such that if $(g, \xi) \in \Gamma$ and $[\xi] \geqq C$, then the following holds: $\pi_{\xi}\left(P_{g}^{0}\right)$ is injective on $\mathscr{S}_{\pi_{\xi}}$ and $\pi_{\eta}\left(P_{g}^{0}\right) \neq 0$ for all $\eta \in \partial \Gamma_{\xi},[\eta] \geqq C$. Then $P$ is $\delta$-microhypoelliptic on $\Gamma$.

Proof. The theorem will be proved first under the assumption that $p \in S^{m}\left(\mathscr{G}^{*}, \delta\right)$, i.e. that $P$ is left invariant. In that case, by Corollary 2.2 we need only consider microhypoellipticity over the identity element $e$ of $G$ and may consider $\Gamma$ to be a subset of $\mathscr{G}^{*}$. Let $\xi_{0} \in \Gamma$ and let $\Gamma_{1}$ be an open $\delta$-cone containing $\xi_{0}, \Gamma_{1} \subset \Gamma$. Let $\Gamma^{\prime}=\cup\left\{\mathcal{O}_{\xi}: \xi \in \Gamma_{1}\right\}$. Define $h(\xi)$ by (1.8).

Lemma 4.2. If $p \in S^{m}\left(\mathscr{G}^{*}, \delta\right)$ satisfies the hypotheses of Theorem 4.1 then there exist $C$ and $c>0$ such that $|p(\xi)| \geqq c \chi(\xi)^{m}$ for all $\xi \in \Gamma^{\prime}$ such that $[\xi] \geqq C$ and $h(\xi) \leqq c$.

Proof. Since $p^{0}(\eta) \neq 0$ for $\eta \in \partial \Gamma_{\xi}, \xi \in \Gamma_{1}, \eta$ large, there exist $C_{1}$ and $c_{1}>0$ such that if $\xi \in \Gamma^{\prime},[\xi]=C_{1}$ and $\left|\xi^{\prime}\right| \leqq c_{1}$, then $\left|p^{0}(\xi)\right| \geqq c_{1}$. As before, $\xi^{\prime}=\left.\xi\right|_{s_{2}}$. There is a $C_{2}$ such that $\left|\delta_{c_{1}} \xi\right| \leqq C_{2}|\xi|$ for all $\xi \in \mathscr{G}^{*}$. Let $c=c_{1} / \max \left\{C_{2}, C_{1}^{m}\right\}$. Given $\xi \in \Gamma$ such that $[\xi] \geqq C_{1}$ and $h(\xi) \leqq c$, let $r=C_{1}^{-1}[\xi]$ and $\xi_{0}=\delta_{r}^{-1} \xi$. Then $\left[\xi_{0}\right]=C_{1}$ and $\left[\xi_{0}^{\prime}\right] \leqq C_{2}\left|\delta_{[\xi]}^{-1} \xi^{\prime}\right|=C_{2} h(\xi) \leqq c_{1}$. Thus $\left|p^{0}(\xi)=r^{m}\right| p^{0}\left(\xi_{0}\right) \mid \geqq c \chi(\xi)$. The lemma follows by choosing $C$ large enough that $\chi(\xi)^{-m}\left|p^{1}(\xi)\right| \leqq c / 2$ if $[\xi] \geqq C$.

A special type of cut-off function, as described in the next lemma, will be needed in dealing with open $G$-invariant $\delta$-cones $\Gamma_{2} \subseteq \Gamma_{1} \subseteq \mathscr{G}$. Note that for such $\Gamma_{1}$ and $\Gamma_{2} \neq 0$ it is impossible to have $\bar{\Gamma}_{2} \subseteq \Gamma_{1}$, since $\bar{\Gamma}_{2} \cap \mathscr{G}^{*} \neq \phi$ by Proposition 3.2. 
Definition. Given a $G$-invariant $\delta$-cone $\Gamma \subseteq \mathscr{G}^{*}$, let $i \Gamma=\left\{(\rho, \zeta) \in R^{N-2 d} \times \mathscr{G}_{2}^{*}: \mathcal{O}_{\rho \zeta} \subset \Gamma\right.$ and $[\zeta]=1\}, \mathcal{O}_{\rho \zeta}$ defined as at the end of Section 1. If $\Gamma_{1}$ and $\Gamma_{2}$ are open $G$-invariant $\delta$ cones such that $\Gamma_{2} \subseteq \Gamma_{1} \subseteq \mathscr{G}^{*}$ and $\left(i \Gamma_{2}\right)^{-}$is a compact subset of $i \Gamma_{1}$, then we write $\Gamma_{2} \subseteq \Gamma_{1}$ properly.

Definition. $S_{00}^{m}\left(\mathscr{G}^{*}, \delta\right)$ is the set of functions $p$ for which estimate (1.6) is required only for derivatives in directions parallel to the orbits of the coadjoint action (see [12]). $S_{0}^{m}\left(\mathscr{G}^{*}, \delta\right)$ is the set of $p$ such that $D^{\alpha} p \in S_{00}^{m}\left(\mathscr{G}^{*}, \delta\right)$ for all $\alpha$ such that $\alpha^{\prime}=\left(\alpha_{N+1}, \ldots, \alpha_{n}\right)=0$. If $\Gamma$ is an open subset of $\mathscr{G}^{*}, S^{m}(\Gamma, \delta)$ is the set of $p \in C^{\infty}(\Gamma)$ such that $p$ has an extension in $S^{M}\left(\mathscr{G}^{*}, \delta\right)$ for some $M$ and such that (1.6) holds for $\xi \in \Gamma$.

Lemma 4.3. Let $\Gamma_{1}$ and $\Gamma_{2}$ be open $G$-invariant $\delta$-cones such that $\Gamma_{1} \subseteq \mathscr{G}^{*}$ and $\Gamma_{2} \subseteq \Gamma_{1}$ properly. Given $c_{1}>c_{2} \geqq 0$, there is $a \varphi \in S_{0}^{0}\left(\mathscr{G}^{*}, \delta\right)$ such that $\operatorname{supp} \varphi \subset \Gamma_{1}, \varphi(\xi)=1$ if $\xi \in \Gamma_{2}$ and $\left[\xi^{\prime}\right] \geqq c_{1}, \varphi(\xi)=0$ if $[\xi] \leqq c_{2}$.

Proof. If $\Gamma$ is a $G$-invariant $\delta$-cone and $\mathcal{O}_{\rho \xi} \subset \Gamma$, then $\mathcal{O}_{\rho, \delta \gamma} \subset \Gamma$ for all $r>0$. Thus one can find a function $\varphi_{0} \in C^{\infty}\left(R^{N-2 d} \times \mathscr{G}_{2}^{*}\right)$ such that $\varphi_{0}(\rho, \zeta)=0$ if $\mathcal{O}_{\rho \zeta} \cap \Gamma_{1}=\phi, \varphi_{0}(\rho, \zeta)=0$ if $[\zeta] \leqq c_{2}, \varphi_{0}(\rho, \zeta)=1$ if $\mathcal{O}_{\rho \zeta} \subset \Gamma_{2}$ and $[\zeta] \geqq c_{1}, \varphi_{0}\left(\rho, \delta_{r} \zeta\right)=\varphi_{0}(\rho, \zeta)$ if $[\zeta] \geqq c_{1}$ and $r \geqq 1$ and $\left|D_{\rho}^{\alpha} \varphi_{0}(\rho, \zeta)\right| \leqq C_{\alpha}$ on $R^{N-2 \alpha} \times \mathscr{G}_{2}^{*}$. Define $\varphi \in C^{\infty}\left(\mathscr{G}^{*}\right)$ so that $\varphi(\xi)=\varphi_{0}(\rho, \zeta)$ if $\pi_{\xi}=\pi_{\rho \zeta}$, $\varphi(\xi)=0$ if $\xi \notin \Gamma_{1}$. Since $\varphi$ is constant on orbits it follows that $\varphi \in S_{0}^{0}\left(\mathscr{G}^{*}, \delta\right)$.

Note that if $\xi \in \Gamma_{1} \subseteq \mathscr{G}^{*}$ with $\Gamma_{1}$ an open $G$-invariant $\delta$-cone, then there is an open $G$ invariant $\delta$-cone $\Gamma_{2}$ such that $\xi \in \Gamma_{2}$ and $\Gamma_{2} \subseteq \Gamma_{1}$ properly. To simplify notation we will, without specific mention, occasionally replace the $G$-invariant $\delta$-cone $\Gamma^{\prime}$, chosen before Lemma 4.2 , by a properly contained $G$-invariant $\delta$-cone, also written $\Gamma^{\prime}$, still containing $\xi_{0}$. If $c>0$, then $\Gamma_{c}^{\prime}=\left\{\xi \in \Gamma^{\prime}:\left[\xi^{\prime}\right]>c\right\}$. The constant $c$ may also change from statement to statement.

Lemma 4.4. If $p \in S^{m}\left(\mathscr{G}^{*}, \delta\right)$ satisfies the hypotheses of Theorem 4.1, then there exist $b \in S_{00}^{-m}\left(\mathscr{G}^{*}, \delta\right)$ and $c>0$ such that $b \bigsqcup p-1$ and $p \bigsqcup b-1$ are in $S_{00}^{0, k}\left(\Gamma_{c}^{\prime}, \delta\right)$ for all $k$.

Proof. Let $c$ and $C$ be as in Lemma 4.2 and $\varphi$ as in Lemma 4.3. Let $F \in C^{\infty}(R)$ satisfy $F(r)=1$ if $r \geqq 2$ and $F(r)=0$ if $r \leqq 1$. Define $b_{0}(\xi)=\varphi(\xi) F\left(C^{-1}[\xi]\right) F\left(\operatorname{ch}(\xi)^{-1}\right) p(\xi)^{-1}$. Then $b_{0} \in S^{-m}\left(\mathscr{G}^{*}, \delta\right)$ and $1-\left(b_{0} \square p\right) \in S_{00}^{0.1}\left(\Gamma_{2 C}^{\prime}, \delta\right)$ by Theorem 1.1. The desired symbol $b$ is now constructed by the standard parametrix method.

Returning to the proof of Theorem 4.1, the injectivity of $\pi_{\xi}\left(P^{0}\right)$ on $\mathscr{S}\left(R^{d}\right)$ implies, by Theorem 7.7 of [1], that $\pi_{\xi}\left(P^{0}\right)$ has an inverse which is a pseudodifferential operator. There is a $c>0$ such that if $[\xi]>c, \xi \in \Gamma^{\prime}$, then $\pi_{\xi}(P)$ also has an inverse which is a pseudodifferential operator, the Weyl symbol of which we denote by $q_{\xi}$. Define $q$ on $\Gamma_{c}^{\prime}$ by $\left.q\right|_{\mathscr{O}_{\xi}}=q_{\xi} \circ \psi_{\xi}^{-1}$, where $\psi_{\xi}: R^{d} \times R^{d} \rightarrow \mathcal{O}_{\xi}$ is the symplectomorphism described in Section 1. Using Lemma 4.4 it follows by arguments given in [13] that $q \in S_{00}^{-m}\left(\Gamma_{c}^{\prime}, \delta\right)$. Replace $q$ by $\varphi q$ for $\varphi$ as in Lemma 4.3. Then $q \in S_{00}^{-m}\left(\mathscr{G}^{*}, \delta\right)$ and $q \square p=1$ on $\Gamma_{c}^{\prime}$

In order to obtain estimates for derivatives transverse the orbits we will look at difference quotients. Given $\tau \in \mathscr{G}^{*}$ and $p \in S_{00}^{m}\left(\mathscr{G}^{*}, \delta\right)$, define $p_{\tau}(\xi)=p(\xi+\tau)$. If $\tau \in \mathscr{G}_{1}^{*}$, then $(p \bigsqcup q)_{\mathrm{r}}=p_{\tau} \bigsqcup q_{\mathrm{r}}$. For general $\tau$ and for $p \in S_{0}^{m}\left(\mathscr{G}^{*}, \delta\right), q \in S_{0}^{k}\left(\mathscr{G}^{*}, \delta\right)$ we use formula (2.2) and Taylor's Theorem to find that

$$
(p \bigsqcup q)_{\mathrm{r}}=p_{\mathrm{r}} \bigsqcup q_{\mathrm{r}}+(2 i)^{-1} B_{\mathrm{r}}^{\natural}\left(p_{\mathrm{r}}, q_{\mathrm{z}}\right)+R_{\mathrm{r}}(p, q)
$$


where

$$
B_{\varepsilon}^{\square}(p, q)(\xi)=i \int e^{i\langle\eta-\xi, y\rangle}\left(\operatorname{ad} y^{*} \tau\right) \cdot \nabla p\left(\xi+\frac{1}{2} \operatorname{ad} y^{*} \xi\right) q(\eta) d y d \eta
$$

and where $\lim _{\mathrm{r} \rightarrow 0}|\tau|^{-1} R_{\mathrm{r}}(p, q)=0$ uniformly on compact subsets of $\mathscr{G}^{*}$. By (1.2) the $j$ th component of ad $y^{*} \tau$ is $\sum \gamma_{l j}^{k} \tau_{k} y_{l}$, and consequently by (2.2)

$$
B_{\tau}^{\natural}(p, q)=\sum \gamma_{j l}^{k} \tau_{k} \partial_{j} p \bigsqcup \partial_{l} q \text {. }
$$

Given $\xi \in \Gamma_{c}^{\prime}$ there is a $t_{0}>0$ such that if $\tau=t e_{k}^{*}$, where $e_{k}^{*}$ is one of the chosen basis vectors for $\mathscr{G}^{*}$ and $|t|<t_{0}$, then

$$
(p \bigsqcup q)_{\tau}(\xi)=p \bigsqcup q(\xi)=1 .
$$

If $k \leqq N=\operatorname{dim} \mathscr{G}_{1}$, then

$$
p \bigsqcup\left(q_{\tau}-q\right)=-\left(p_{\tau}-p\right) \bigsqcup q_{\tau} \text {. }
$$

Without loss of generality we may assume that $\pi_{\xi}(Q)$ is a two sided inverse for $\pi_{\xi}(P)$, for if not $P$ can be multiplied on the left by its adjoint. Therefore, $q_{\mathrm{t}}-q=-q \square\left(p_{\mathrm{r}}-p\right) \square q_{\mathrm{t}}$ and hence $D_{k} q=-q \square D_{k} p \square q \in S_{00}^{-m-\mu_{k}}\left(\Gamma_{c}^{\prime}, \delta\right)$. Proceeding by induction on $|\alpha|$, we find that $D^{\alpha} q \in S_{00}^{-m-\mu \alpha}\left(\Gamma_{c}^{\prime}, \delta\right)$ for all $\alpha$ such that $\alpha^{\prime}=0$. Multiplying $q$ by a cut-off function as in Lemma 4.3 we may also assume that $q \in S_{0}^{-m}\left(\mathscr{G}^{*}, \delta\right)$.

Similarly, for $k>N$ we use (4.1) to find that $D_{k} q(\xi)$ exists for $\xi \in \Gamma_{c}^{\prime}$ and

$$
D_{k} q=-q \square D_{k} p \square q+\frac{1}{2} q \bigsqcup B_{k}^{\natural}(p, q)
$$

where $B_{k}^{\natural}=B_{\tau}^{\natural}$ for $\tau=e_{k}^{*}$. By (1.3), (4.2) and (4.3) we find that

$$
\left|D_{k} q(\xi)\right|<C \chi(\xi)^{-m-\mu_{k}}, \quad \xi \in \Gamma_{c}^{\prime}
$$

By an inductive argument $q \in S^{-m}\left(\Gamma_{c}^{\prime}, \delta\right)$. Multiplying $q$ by a cut-off function $\varphi \in S^{0}\left(\mathscr{G}^{*}, \delta\right)$ which is 1 on a $\delta$-cone containing $\xi_{0}$, the proof of Theorem 4.1 for left invariant $P$ now follows from Propositions 2.4 and 2.6.

Turning to the proof of the theorem for non-invariant $P$, let $\left(g_{0}, \xi_{0}\right) \in \Gamma$. As noted in Section 2 there is a neighborhood $\omega_{1}$ of $g_{0}$ and an open $\delta$-cone $\Gamma_{1}$ containing $\xi_{1}=\operatorname{Ad} \frac{1}{2} g_{0}^{*} \xi_{0}$ such that $\omega_{1}^{*} \Gamma_{1} \subset \Gamma$. If $\eta \in \Gamma_{1}$ and $g \in \omega_{1}$, then $\eta=\operatorname{Ad} \frac{1}{2} g^{*} \xi \in \mathcal{O}_{\xi}$ for some $\xi$ such that $(g, \xi) \in \Gamma$ and therefore $\pi_{\eta}\left(P_{g}^{0}\right) \cong \pi_{\xi}\left(P_{g}^{0}\right)$ is injective and $\pi_{\eta}\left(P_{g}^{0}\right) \neq 0$ for all $\bar{\eta} \in \partial \Gamma_{\eta}=\partial \Gamma_{\xi},[\bar{\eta}] \geqq C$. The theorem having been proved in the invariant case, for each $g \in \omega_{1}$ there is a $q_{g}^{0}$ such that $q_{g}^{0} \square p_{\theta}=1$ for all $\eta \in \Gamma_{1}$ such that $[\eta] \geqq C$. Define $q^{0}(g, \xi)=\varphi(g) q_{g}^{0}(\xi)$ where $\varphi \in C_{0}^{\infty}\left(\omega_{1}\right), \varphi=1$ on a neighborhood $\omega_{2}$ of $g_{0}$. Let $r=1-q^{0} \square p$. Then $q^{0} \in S^{-m}\left(\Omega \times \mathscr{G}^{*}, \delta\right)$ and $r \in S^{-M}\left(\omega_{2} \times \Gamma_{1}, \delta\right)$ for all $M$, the appropriate estimates for derivatives along $G$ being established by the same methods as above. For positive integers $k$ define

$$
q^{k}=-\sum_{0<|\alpha| \leqq k}(\alpha !)^{-1} \partial_{\xi}^{\alpha} q^{k-|\alpha|} \bigsqcup T_{g}^{\alpha} p \bigsqcup q^{0}
$$


and chooses $q \in S^{-m}\left(\Omega \times \mathscr{G}^{*}, \delta\right)$ such that $q \sim \sum_{k=0}^{\infty} q^{k}$. Using Theorem 1.2 it follows by standard arguments that $q \# p-1 \in S^{-M}\left(\omega_{2} \times \Gamma_{1}, \delta\right)$ for all $M$. Thus $P$ is $\delta$ microhypoelliptic in a neighborhood of $\left(g_{0}, \xi_{0}\right)$.

Corollary 4.5. Let $\delta=\left\{\delta_{r}: r>0\right\}$ be natural dilations on a step two nilpotent Lie group $G$, i.e. $\delta_{r} x=r^{2} x$ for $x \in \mathscr{G}_{2}, \delta_{r} x=r x$ for $x \in \mathscr{G}_{1}$. Let $p \in S^{m}\left(\Omega \times \mathscr{G}^{*}, \delta\right)$ have principal symbol $p^{0}$. Let $\left(g_{0}, \xi_{0}\right) \in \Omega \times \widetilde{G}^{*}$. If $\pi_{\xi_{0}}\left(P_{g_{0}}^{0}\right)$ is injective and $\pi_{\eta}\left(P_{g_{0}}^{0}\right) \neq 0$ for all $\eta \in R_{\xi_{0}}^{\perp}$ such that $[\eta] \geqq C$, then $P$ is $\delta$-microhypoelliptic in a $\delta$-conic neighborhood of $\left(g_{0}, \xi_{0}\right)$.

Proof. For $\xi \in \mathscr{G}^{*}$ let $\chi_{\xi}=\chi \circ \psi_{\xi}, \psi_{\xi}$ as is Section 1 , and let $H_{\xi}^{m}\left(R^{d}\right)$ be the Sobolev space as defined in [1] corresponding to the weight functions $\Phi=\chi_{\xi}, \varphi=1$ and order $m \log \chi_{\xi}$. If $\xi$ is near $\xi_{0}$, then $\chi_{\xi} \approx \chi_{\xi_{0}}$ and hence $H_{\xi}^{m}=H_{\xi_{0}}^{m}$. By using the Mean Value Theorem and a simple perturbation argument one sees that there is a neighborhood $U$ of $\left(g_{0}, \xi_{0}\right)$ such that $\pi_{\xi}\left(P_{g}^{0}\right): H_{\xi}^{m}\left(R^{d}\right) \rightarrow L^{2}\left(R^{d}\right)$ is injective for all $(g, \xi) \in U$. Furthermore, since the subspace $R_{\xi}$ depends continuously on $\xi$ for $\xi \in \mathscr{G}^{*}$, there is a neighborhood $U$ of $\left(g_{0}, \xi_{0}\right)$ such that $\pi_{\eta}\left(P_{g}^{0}\right) \neq 0$ for all $(g, \xi) \in U, \eta \in R_{\xi}^{\perp},[\eta] \geqq C$. Hence the corollary follows from Theorem 4.1 and Corollary 3.4 .

The proof of the corollary breaks down for arbitrary dilations since $\chi_{\xi}$ is not necessarily equivalent to $\chi_{\xi_{0}}$ for $\xi$ near $\xi_{0}$. Furthermore, $\partial \Gamma_{\xi}$ does not necessarily depend continuously on $\xi \in \tilde{\mathscr{G}}^{*}$, as mentioned at the end of Section 3.

The next corollary gives a sufficient condition for microhypoellipticity in the standard sense. For simplicity the result will be stated for left invariant operators only. A subset $\Lambda \subset \dot{G}^{*}$ is a cone if $\xi \in \Lambda$ implies $r \xi \in \Lambda$ for all $r>0$. Define the standard wave front set $W F(u)$ of a distribution as in [5] or [20] and define microhypoellipticity analogously. If $P \in \mathrm{Op} S^{m}\left(G^{*}, \delta\right)$ and $\pi_{\xi}\left(P^{0}\right)$ is injective for all $\xi \in \Lambda$, then $\pi_{\eta}\left(P^{0}\right)$ is automatically injective for all $\eta$ in the smallest $G$-invariant $\delta$-cone $\Gamma^{\prime}$ containing $\Lambda$. In order to apply the parametrix construction of Theorem 4.1 we will need to assume injectivity on a $\delta$-cone $\Gamma$ containing $\Gamma^{\prime}$ properly.

Corollary 4.6. Let $p \in S^{m}\left(\mathscr{G}^{*}, \delta\right)$ have principal symbol $p^{0}$. Let $\Gamma \in \mathscr{G}^{*}$ be a G-invariant $\delta$-cone such that for $\xi \in \Gamma$ and all $\eta \in \bar{\Gamma}_{\xi}, \pi_{\eta}\left(P^{0}\right)$ is injective. Let $\Lambda$ be an open standard cone such that $\Lambda \subseteq \Gamma^{\prime}$ where $\Gamma^{\prime}$ is a G-invariant $\delta$-cone with $\Gamma^{\prime} \subseteq \Gamma$ properly. Then $P$ is microhypoelliptic on $\Lambda$.

Proof. By the construction in the proof of Theorem 4.1 we obtain $q \in S^{-m}\left(\Gamma_{c}^{\prime}, \delta\right) \cap S_{0}^{-m}\left(\mathscr{G}^{*}, \delta\right)$ with $q \square p=1$ on $\Gamma_{c}^{\prime}$. There is a $C_{1}$ such that if $\xi \in \Lambda$ and $|\xi| \geqq C_{1}$, then $\left[\xi^{\prime}\right] \geqq C$. Given $\xi_{0} \in \Lambda$ there is a function $\varphi \in C^{\infty}\left(\mathscr{G}^{*}\right)$ and a conic neighborhood $\Lambda_{1}$ of $\xi_{0}$ such that $\varphi(\xi)=1$ if $\xi \in \Lambda_{1}$ and $|\xi| \geqq 2 C_{1}$, supp $\varphi \subset \Gamma_{c}^{\prime}$, $\varphi(r \xi)=\varphi(\xi)$ if $|\xi| \geqq 2 C_{1}$ and $r \geqq 1$. Let $q^{\prime}=\varphi q$. Then $Q^{\prime} \in \mathrm{Op} S_{\rho, 1-\rho}^{-m}$ with $\rho=1 / \bar{\mu}$, and $Q^{\prime} P-I$ is regularizing on $\Lambda_{1}$. Since $Q \in \mathrm{Op} S_{\rho, 1-\rho}^{-m}$, it follows from Theorem VI.1.6 of [20] that $W F\left(Q^{\prime} P u\right) \subseteq W F(P u)$. Thus $P$ is microhypoelliptic on $\Lambda$.

As a simple example, let $\mathscr{G}$ be the Heisenberg algebra with basis vectors satisfying $\left[e_{j}, e_{j+n}\right]=e_{2 n+1}$ if $j \leqq n$. Let $\Lambda=\Gamma^{\prime}=\Gamma=\left\{\xi: \xi_{2 n+1}>0\right\}$. Let $\xi_{0}=(0, \ldots, 0,1)$. If $\pi_{\xi_{0}}\left(P^{0}\right)$ is injective and $\pi_{\eta}\left(P^{v}\right) \neq 0$ for all $\eta \in \mathscr{G}_{1}^{*}$, then $P$ is microhypoelliptic on $\Lambda$. For example, if $p(\xi)=\xi_{1}^{2}+\cdots+\xi_{2 n}^{2}+\xi_{2 n+1}$, then $O p(p)$ is microhypoelliptic on $\Lambda$, but is not hypoelliptic on $G$. 


\section{REFERENCES}

1. R. BEALS, A general calculus of pseudodifferential operators, Duke Math J. 42 (1975), 1-42.

2. R. Beals, Operateurs invariants hypoelliptiques sur un groupe de Lie nilpotent (Seminaire Goulaouic-Schwartz, 1976-77, exposé 19), 1-8.

3. A. Grigis, Propagation des singularités sur des groupes de Lie nilpotents de rang 2. II, Ann. Sci. École Norm. Sup. 15 (1982), 161-171.

4. B. Helffer and J. Nourrigat, Caractérization des opérateurs hypoelliptiques homogènes invariants a gauche sur un groupe de Lie nilpotent gradué, Comm. Partial Differential Equations 4, (1979), 899-958.

5. L. Hórmander, The Analysis of Linear Partial Differential Operators I (Springer-Verlag, Berlin and New York, 1983).

6. L. Hormander, The Weyl calculus of pseudo-differential operators, Comm. Pure Appl. Math. 32 (1979), 359-443.

7. A. KIRILLov, Unitary representations of nilpotent Lie groups, Uspekhi Mat. Nauk 17 (1962), no. 4 (106), 57-110; Russian Math. Surveys 17 (1962), 53-104.

8. R. Lascar, Propagation des singularités des solutions d'equations pseudo-differentielles quasihomogènes, Ann. Inst. Fourier (Grenoble) 27 (1977), 79-153.

9. A. MeLin, Parametrix constructions for some classes of right-invariant differential operators on the Heisenberg group, Comm. Partial Differential Equations 6 (1981), 1363-1405.

10. A. Melin, Parametrix Constructions for Right-Invariant Differential Operators on Nilpotent Groups, Ann. Global Anal. Geom. 1 (1983), 79-130.

11. K. Miller, Parametrices for hypoelliptic operators on step two nilpotent Lie groups, Comm. Partial Differential Equations 5 (1980), 1153-1184.

12. K. MilleR, Invariant pseudodifferential operators on two step nilpotent Lie groups, Michigan Math. J. 29 (1982), 315-328; II, Michigan Math. J. 33 (1986), 395-401.

13. K. Miller, Inverses and parametrices for right-invariant pseudodifferential operators on two-step nilpotent Lie groups, Trans. Amer. Math. Soc. 280 (1983), 721-736.

14. K. Miller, Microhypoellipticity on step two nilpotent Lie groups, Contemp. Math. 27 (1984), 231-235.

15. C. Parenti and L. Rodino, Parametrices for a class of pseudo differential operators I, II, Ann. Mat. Pura Appl. 125 (1980), 221-278.

16. C. PARENTI and L. Rodino, Examples of hypoelliptic operators which are not microhypoelliptic, Boll. Un. Mat. Ital. B17 (1980), 390-409.

17. D. Phong and E. Stein, Some further classes of pseudodifferential operators and singularintegral operators arising in boundary-value problems I: Composition of operators, Amer. J. Math 104 (1982), 141-172.

18. C. RockLAND, Hypoellipticity on the Heisenberg group: representation-theoretic criteria, Trans. Amer. Math. Soc. 240 (1978), 1-52.

19. M. TAYLOR, Noncommutative microlocal analysis Part I, Mem. Amer. Math. Soc. 313 (1984).

20. M. TAYLoR, Pseudodifferential Operators (Princeton University Press, Princeton, 1981).

Wichita State University

WICHITA, KS 67208 